\title{
Uveitis in Miller Fisher Syndrome-A Case Report and Literature Review
}

\author{
Jia Jeane Ngai \\ Department of Ophthalmology, Hospital Bintulu, Bintulu, Malaysia \\ Email: jeanengai@gmail.com
}

How to cite this paper: Ngai, J.J. (2017) Uveitis in Miller Fisher Syndrome-A Case Report and Literature Review. Case Reports in Clinical Medicine, 6, 317-323. https://doi.org/10.4236/crcm.2017.612038

Received: November 25, 2017 Accepted: December 26, 2017 Published: December 29, 2017

Copyright $\odot 2017$ by author and Scientific Research Publishing Inc. This work is licensed under the Creative Commons Attribution International License (CC BY 4.0).

http://creativecommons.org/licenses/by/4.0/

\begin{abstract}
Purpose: To report an unusual case of Miller Fisher Syndrome (MFS) in which the patient presented with concurrent right eye uveitis. Case Report: We report a case of a 51-year-old gentleman who presented with typical clinical features of Miller Fisher syndrome including ophthalmoplegia, ataxia, areflexia, ptosis and diplopia following an upper respiratory tract infection. Concurrently, he also had right eye uveitis with raised intraocular pressure. The patient was treated with intravenous immunoglobulin (IVIG), topical steroids and anti-glaucoma eye drops in which he demonstrated good recovery. For diagnostic confirmation, serum antiganglioside antibodies (antiGQ1b IgG) were later reported to be positive. Conclusion: We described the possible association between MFS and uveitis due to its interrelated pathogenesis. This possible association can lead to early detection and treatment of uveitis.
\end{abstract}

\section{Keywords}

Miller Fisher Syndrome, Uveitis, Anti-Ganglioside Antibody, Myasthenia Gravis

\section{Introduction}

Miller Fisher syndrome (MFS) is a rare immune mediated neuropathy [1]. In 1956, Charles Miller Fisher reported MFS as a variant of Guillain-Barré Syndrome (GBS), which was first described in 1932 by James Collier [2]. It was based on 3 observed cases characterized by clinical trial of total external ophthalmoplegia, ataxia, and hyporeflexia [2]. The recognition of this disease is important as the presenting neuro-ophthalmological symptoms are often alarming but the course is generally benign [1] [2]. During our literature search, we found no reported association between MFS and uveitis. However, there were little 
published reports describing GBS and uveitis [3] [4]. Here, we report an unusual case of MFS in which the patient presented with concurrent right eye uveitis and raised intraocular pressure.

\section{Case Report}

A 51 year old gentleman with no significant medical history presented with acute onset of ptosis, binocular horizontal diplopia and poor balance. He attributed his poor balance due to the diplopia and dizziness. He also reported 2 days history of right eye redness and blurring of vision. He has been having fever, rhinorrhoea and cough for the past 2 weeks but was beginning to subside. $\mathrm{He}$ did not complain of shortness of breath or difficulty in speech and swallowing. He had no previous episodes of blurring of vision or eye redness.

On ophthalmic examination, he had bilateral ptosis (see Figure 1) with restricted movements of horizontal and upward gaze. Corrected visual acuity were $6 / 15$ over both eyes. Right pupil was $6 \mathrm{~mm}$ and unreactive while the left pupil was $4 \mathrm{~mm}$ with sluggish reaction. There was no nystagmus observed. Right eye showed features of anterior uveitis with raised intraocular pressure of $28 \mathrm{mmHg}$ as compared to the left intraocular pressure of $18 \mathrm{mmHg}$. Right eye cornea was hazy and oedematous with absence of keratic precipitates. Anterior chamber was deep with $2+$ cells and conjunctiva was hyperemic. There was absence of anterior or posterior synechiae. Left eye anterior segment was normal. Both eyes had immature nuclear cataract. Gonioscopy of both eyes revealed open angles. Fundus visualization over the right eye was poor while the left eye had normal fundus. B-scan ultrasonography showed no vitritis.

Same day evaluation by the medical team noted his limbs power were full with intact deep tendon reflexes. Sensory examinations were intact. Barbinski's sign was negative. Other cranial nerves examination were unremarkable. Romberg's test was negative. He had no wide based gait but was unable to perform the tandem gait test. No past pointing and no dysdiadochokinesia. Examinations of other systems were normal.

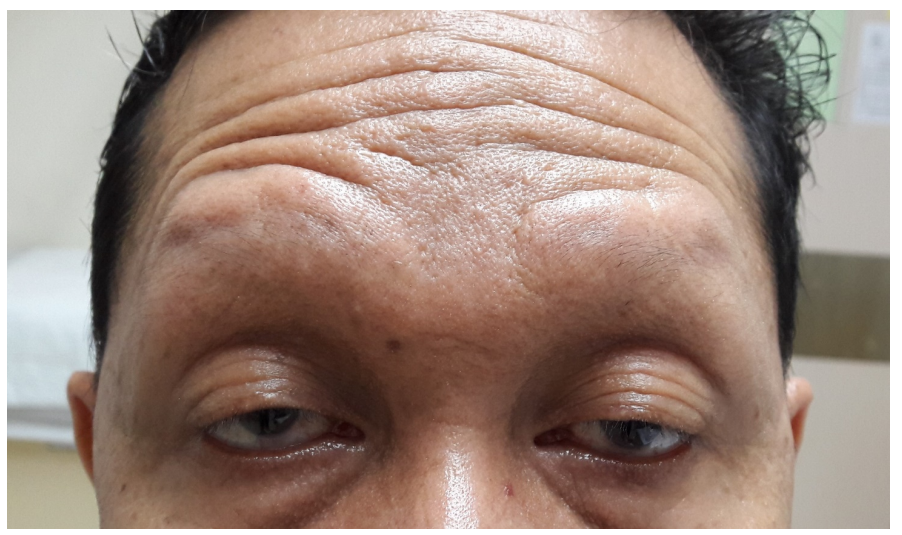

Figure 1. The patient had marked bilateral ptosis with wrinkling of the forehead and total external ophthalmoplegia. 
Routine blood and infectious screening results were normal. These included complete blood count, renal function test, liver function test, fasting lipid profile, fasting blood glucose, serum HIV antibody, HepBsAg, serum Hepatitis C antibody and serum VDRL. No significant finding on chest radiograph and CT brain. Initial working diagnosis was ocular myasthenia gravis and patient was started on pyridostigmine.

During the following day of admission, he had bilateral complete external ophthalmoplegia and areflexia. A diagnosis of MFS was considered based on clinical grounds while awaiting for confirmation result of serum antiganglioside antibodies. Lumbar puncture was performed prior to initiation of intravenous immunoglobulin (IVIG). Opening pressure was normal and CSF analysis showed elevated protein of $0.56 / \mathrm{L}$ with total cell of 1 perc.m.m. CSF for cytology, bacterial antigen, AFB staining, culture and sensitivity were negative. He completed a 5 days course of IVIG $0.4 \mathrm{~g} / \mathrm{kg} /$ day. His ophthalmoplegia and gait gradually improved. At the same time, he was started on topical steroids (Betamethasone $0.1 \%$ ) and anti-glaucoma eye drops (Latanoprost $0.005 \%$ and Timolol $0.5 \%)$ which were gradually tapered over a period of a month. Right eye uveitis subsided and intraocular pressure was normalized. For diagnostic confirmation, serum antiganglioside antibodies (anti-GQ1b IgG) was later reported to be positive while acetylcholine receptor antibodies were negative. During follow up of the patient after 2 months, we noted complete resolution of his ophthalmoplegia and unsteady gait.

\section{Discussion}

MFS accounts to $5 \%$ to $10 \%$ of GBS cases in the Western population. In contrast, the incidence appears to be higher in the Asian population, with 19\% of GBS cases in Taiwan and 25\% in Japan [5] [6] [7]. As the reason for this discrepancy has not been established, environmental factors may possibly play a role [8]. MFS can occur in all age groups with median age of onset at 40 years. It occurs twice as commonly in men than women [5] [9]. Peak incidence was noted during the Spring season [5].

Chiba et al in 1992 proposed anti-GQ1b IgG antibody as a diagnostic marker of MFS [10]. A study by Ito et al on 466 MFS patients identified that $83 \%$ were tested positive for serum anti-GQ1b IgG antibody [11]. Majority of MFS is preceded by infection prior to onset of their neuro-ophthalmological symptoms, which justify the hypotheses of MFS being a postinfectious neuroimmune disease [5] [9]. A study looking at evidence of antecedent infections such as Campylobacter jejuni, Cytomegalovirus, Epstein-Barr virus, Mycoplasma pneumoniae, and Haemophilus influenzae found serological isolation of $C$. jejuni and Haemophilus influenza in MFS [12]. In another study, $C$. jejuni, a gram negative bacteria which often causes enteritis, also found to be the most common organism associated with MFS [11]. In most MFS patients with antecedent $C$. jejuni or Haemophilus influenza infection, anti-GQ1b IgG antibody was detected [12]. There was also a reported case of anti-GQ1b antibodies found in MFS related to 
Epstein-Barr virus [13].

GQ1b ganglioside is concentrated in oculomotor, trochlear, abducens nerves and group Ia afferents in muscle spindles [14] [15]. The hypothesised pathogenesis of MFS is that production of anti-GQ1b antibodies mediated by the molecularly similar lipo-oligosaccharides on the surface of preceding infectious agent bind to the GQ1b ganglioside [12]. This accounts for high incidence of ophthalmoplegia and ataxia [8] [16].

MFS is fundamentally a clinical diagnosis, as described in 1956 [2]. Initial presenting symptoms were diplopia and ataxia. $81.6 \%$ had areflexia. Some had other cranial nerves involvement, most commonly cranial nerve VII, followed by cranial nerve IX, X and XII. The typical presentation of ophthalmoplegia is symmetrical upgaze palsy followed by horizontal gaze palsy and lastly downgaze palsy [17]. Diplopia arises due to the presence of external ophthalmoplegia [1]. Aside from the classical triad of total external ophthalmoplegia, ataxia, and hyporeflexia, other clinical features in MFS were also described. Incidence of blepharoptosis, variable pupillary dysfunction (internal ophthalmoplegia) or facial palsy were frequently found [5]. However, sensory loss was infrequent and disproportional to the gross ataxia [5]. MFS as compared to GBS, rarely manifest respiratory or cardiac complications that requires ventilator support [18].

During the first encounter with patient presenting with acute brainstem syndrome such as ophthalmoloplegia, pupillary abmormalities and ataxia, it is important to exclude life threatening causes such as brainstem stroke. Neuroimaging like CT or preferably MRI brain are helpful in detection of vascular, infiltrative, or demyelinating disorder [8]. Onset of ophthalmoplegia in MFS is often rapid as compared to other diseases such myotonic dystrophy, ocular my-asthenia gravis (MG), thyroid eye disease, which progress more gradually [1].

Ocular MG often mimics MFS as both produce similar ophthalmic signs during the initial presentation. Both condition can present with external ophthalmoplegia, variable ptosis and diplopia. In MG, ptosis and diplopia worsens by the end of the day, whereas in MFS remains the same as the ophthalmoplegia is often complete and bilateral [1]. However, MG has not been known to cause internal ophthalmoplegia, ataxia or areflexia.

In this case, ocular MG was the early working diagnosis due to patient's initial presentation and its more common occurrences compared to MFS. His first presentation to us was incomplete external ophthalmoplegia, ptosis and diplopia with intact deep tendon reflexes. However, just over a course of a day, his external ophthalmoplegia became complete and we were unable to elicit the deep tendon reflexes. The fact that patient also had ataxia and pupillary abnormality prompted us to an alternative diagnosis of MFS. CSF analysis in MFS can be normal, mild pleocytosis or have an albuminocytological dissociation [9]. The patient's CSF analysis showed an albuminocytological dissociation. Lastly, the most specific test to support the diagnosis of MFS is presence of anti GQ1b IgG antibodies.

MFS generally follows a benign course of recovery. Ataxia and ophthalmople- 
gia showed improvements starting 2 weeks after onset of symptoms with mean recovery within 10 weeks [5] [9]. However, only one-third had recovered from areflexia or hyporeflexia. Most patients had little or no disability and were able to return to their normal activities within 6 months after symptoms onset [9]. Mori et al also reported that sex, age, prior infection, complete or in-complete ophthalmoplegia during peak of illness or period of latency to peak of less than 1 week had no association with chances of recovery [9]. MFS can rarely recur but its recurrence was found to be higher as compared to GBS. Clinical manifestations during relapses were identical to typical MFS and is less severe [19] [20].

There are no available clinical trials yet in supporting basis of treatment for MFS [6]. Mode of treatment such as plasmapheresis and intravenous immunoglobulin (IVIG) had been used but showed no beneficial effect in the end outcomes. On one hand, IVIG only slightly fasten improvement of ophthalmoplegia and ataxia but complete recovery remained similar with those without treatment [21]. On the other hand, plasmapharesis shown no clinically signif-icant benefits in typical MFS. This is presumably due to the self-limiting nature of MFS [22].

Diagnosis of uveitis is often presumptive as it is difficult to be proved by culture or serology. Although aqueous and vitreous sampling are relatively safe, cultures of these samples are rarely positive [23]. It is also not routinely done especially in those who responded well to treatment. Serological test for toxoplasma, CMV, HVS is of limited significance due to the high prevalence of general population having these antibodies [23].

Direct correlation between MFS and uveitis has not been established. There were little reported cases of GBS and uveitis [3] [4]. The exact mechanism on how uveitis is linked to GBS is still unclear. Autoimmune disorders or immune-mediated diseases are linked to development of uveitis. Autoimmune disorder is believed to be driven by abnormal immune recognition of self, whilst immune mediated is generally an innate inflammatory reaction triggered by environmental factors [24]. Antecedent infection in the process of MFS could raise the possibility of the patient developing uveitis. There were some reports indicating that common preceding pathogen in MFS including C. jejuni, Haemophilus influenza, Epstein-Barr virus can be associated with uveitis [25] [26] [27]. Furthermore, uveitis has been closely associated with neurological diseases. In a report by Smith et al showed about $8 \%$ of uveitis cases had associated neurological symptoms [28].

\section{Conclusion}

MFS is a rare acquired nerve disease and a great proportion of our current understanding of the disease is based on serial case reports. Further reporting of this association and investigations are required to determine if uveitis is associated with MFS. This case illustrates the possible association between MFS and uveitis which can lead to early detection and treatment of uveitis to achieve a better visual outcome. We recommend a co-management between neurology and ophthalmology department in cases of MFS. 


\section{References}

[1] Anthony, S., Thurtell, M. and Leigh, R. (2012) Miller Fisher Syndrome Mimicking Ocular Myasthenia Gravis. Optometry and Vision Science, 89, e118-e123. https://doi.org/10.1097/OPX.0b013e31827717c1

[2] Fisher, M. (1956) An Unusual Variant of Acute Idiopathic Polyneuritis (Syndrome of Ophthalmoplegia, Ataxia and Areflexia). New England Journal of Medicine, 255, 57-65. https://doi.org/10.1056/NEJM195607122550201

[3] Walker, E. (1990) Guillain-Barré Syndrome and Pan-Uveitis. Scottish Medical Journal, 35, 22. https://doi.org/10.1177/003693309003500109

[4] Maca, S., Scharitzer, M. and Barisani-Asenbauer, T. (2006) Uveitis and Neurologic Diseases: An Often Overlooked Relationship. Wiener Klinische Wochenschrift, 118, 273-279. https://doi.org/10.1007/s00508-006-0601-6

[5] Mori, M., Kuwabara, S., Fukutake, T., Yuki, N. and Hattori, T. (2001) Clinical Features and Prognosis of Miller Fisher Syndrome. Neurology, 56, 1104-1106. https://doi.org/10.1212/WNL.56.8.1104

[6] Overell, J.R., Hsieh, S.T., Yuki, N. and Willison, H.J. (2007) Treatment for Fisher Syndrome, Bickerstaffs Brainstem Encephalitis and Related Disorders. The Cochrane Database of Systematic Reviews.

[7] Lyu, R.-K., Tang, L.-M., Cheng, S.-Y., et al. (1997) Guillain-Barré Syndrome in Taiwan: A Clinical Study of 167 Patients. Journal of Neurology, Neurosurgery, and Psychiatry, 63, 494-500. https://doi.org/10.1136/jnnp.63.4.494

[8] Shahrizaila, N. and Yuki, N. (2012) Bickerstaff Brainstem Encephalitis and Fisher Syndrome: Anti-GQ1b Antibody Syndrome. Journal of Neurology, Neurosurgery \& Psychiatry, 84, 576-583. https://doi.org/10.1136/jnnp-2012-302824

[9] Berlit, P. and Rakicky, J. (1992) The Miller Fisher syndrome. Review of the Literature. Journal of Clinical Neuro-Ophthalmology, 12, 57-63.

[10] Chiba, A., Kusunoki, S., Shimizu, T. and Kanazawa, I. (1992) Serum IgG Antibody to Ganglioside GQ1b Is a Possible Marker of Miller Fisher Syndrome. Annals of Neurology, 31, 677-679. https://doi.org/10.1002/ana.410310619

[11] Ito, M., Kuwabara, S., Odaka, M., Misawa, S., Koga, M., Hirata, K., et al. (2008) Bickerstaff s Brainstem Encephalitis and Fisher Syndrome form a Continuous Spectrum. Journal of Neurology, 255, 674-682.

https://doi.org/10.1007/s00415-008-0775-0

[12] Koga, M., Gilbert, M., Li, J., Koike, S., Takahashi, M., Furukawa, K., et al. (2005) Antecedent Infections in Fisher Syndrome: A Common Pathogenesis of Molecular Mimicry. Neurology, 64, 1605-1611. https://doi.org/10.1212/01.WNL.0000160399.08456.7C

[13] Schnorf, H., Rathgeb, J.P. and Kohler, A. (1998) Anti-GQ1b-Positive Miller Fisher Syndrome in a Patient with Acute Epstein-Barr Virus Infection and Negative Campylobacter Serology. European Neurology, 40, 177.

[14] Chiba, A., Kusunoki, S., Obata, H., Machinami, R. and Kanazawa, I. (1993) Serum Anti-GQ1b IgG Antibody Is Associated with Ophthalmoplegia in Miller Fisher Syndrome and Guillain-Barre Syndrome: Clinical and Immunohistochemical Studies. Neurology, 43, 1911. https://doi.org/10.1212/WNL.43.10.1911

[15] Liu, J., Willison, H. and Pedrosa-Domellöf, F. (2009) Immunolocalization of GQ1b and Related Gangliosides in Human Extraocular Neuromuscular Junctions and Muscle Spindles. Investigative Opthalmology \& Visual Science, 50, 3226. https://doi.org/10.1167/iovs.08-3333 
[16] Kuwabara, S., Asahina, M., Nakajima, M., Mori, M., Fukutake, T., Hattori, T., et al. (1999) Special Sensory Ataxia in Miller Fisher Syndrome Detected by Postural Body Sway Analysis. Annals of Neurology, 45, 533-536. https://doi.org/10.1002/1531-8249(199904)45:4<533::AID-ANA19>3.0.CO;2-H

[17] Al-Din, N., Anderson, M., Eeg-Olofsson, O., et al. (1994) Neuro-Ophthalmic Manifestations of the Syndrome of Ophthalmoplegia, Ataxia, and Areflexia: A Review. Acta Neurologica Scandinavica, 89, 157-163. https://doi.org/10.1111/j.1600-0404.1994.tb01654.x

[18] Lo, Y.L. (2007) Clinical and Immunological Spectrum of the Miller Fisher Syndrome. Muscle \& Nerve, 36, 615-627. https://doi.org/10.1002/mus.20835

[19] Chida, K., Nomura, H., Konno, H., Takase, S. and Itoyama, Y. (1999) Recurrent Miller Fisher Syndrome: Clinical and Laboratory Features and HLA Antigens. Journal of the Neurological Sciences, 165, 139-143. https://doi.org/10.1016/S0022-510X(99)00095-7

[20] Ishii, J., Yuki, N., Kawamoto, M., Yoshimura, H., Kusunoki, S. and Kohara, N. (2016) Recurrent Guillain-Barré Syndrome, Miller Fisher Syndrome and Bickerstaff Brainstem Encephalitis. Journal of the Neurological Sciences, 364, 59-64. https://doi.org/10.1016/j.jns.2016.03.008

[21] Mori, M., Kuwabara, S., Fukutake, T. and Hattori, T. (2007) Intravenous Immunoglobulin Therapy for Miller Fisher Syndrome. Neurology, 68, 1144-1146. https://doi.org/10.1212/01.wnl.0000258673.31824.61

[22] Mori, M. (2002) Plasmapheresis and Miller Fisher Syndrome: Analysis of 50 Consecutive Cases. Journal of Neurology, Neurosurgery \& Psychiatry, 72, 680-680. https://doi.org/10.1136/jnnp.72.5.680

[23] Mustafa, M., Muthusamy, P., Hussain, S., Shimmi, S. and Sein, M. (2014) Uveitis: Pathogenesis, Clinical Presentations and Treatment. IOSR Journal of Pharmacy, 4, 42-47. https://doi.org/10.9790/3013-0401201042047

[24] Caspi, R. (2010) A Look at Autoimmunity and Inflammation in the Eye. Journal of Clinical Investigation, 120, 3073-3083. https://doi.org/10.1172/JCI42440

[25] Howard, R., Sarkies, N. and Sanders, M. (1987) Anterior Uveitis Associated with Campylobacter Jejuni Infection. Journal of Infection, 14, 186-187. https://doi.org/10.1016/S0163-4453(87)92102-5

[26] Sakai, J., Usui, M. and Usui, N. (1990) The Possible Association of Epstein-Barr Virus and Uveitis. Nippon Ganka Gakkai Zasshi, 94, 496-507.

[27] Hannu, T., Sihto-Kauppi, K., Kotaniemi, K. and Kauppi, M. (2004) Acute Anterior Uveitis in Association with an Outbreak of Campylobacter jejuni Infection. Scandinavian Journal of Rheumatology, 33, 55-57. https://doi.org/10.1080/03009740310004045

[28] Smith, J. (2004) Neurological Concomitants of Uveitis. British Journal of Ophthalmology, 88, 1498-1499. https://doi.org/10.1136/bjo.2003.040519 